\title{
Survey on RedTacton: An Innovative Human Area Networking Technology
}

\author{
Rahul Shirbhate $^{1}$, Vishal Mogal ${ }^{2}$ \\ ${ }^{1}$ M.E., Dept. of Computer, RMD Sinhgad School of Engineering, Pune, India \\ ${ }^{2}$ Assistant Professor, Dept. of Computer, RMD Sinhgad School of Engineering, Pune, India
}

\begin{abstract}
We all are well aware of types of network i.e. LAN, MAN and WAN, but if this network is shifted towards human body, means if the network lies within the human body then such type of network is called as Human Area Network(HAN). This paper describes a new era of technology, a technology we call Red Tacton. For transfer of the data, Red Tacton uses human body as a medium. It uses IEEE 802.3 standard to have a data rate of up to 10Mbps. Its transmitter uses the bodys minute electrical field to transmit messages in the form of digital signal. The human body send most of the electricity to the receiver, as it senses, Voltage change in the electric field, the receivers decode them to receive the data.
\end{abstract}

Keywords: Red Tacton, Bodys Electrical Field, Transmitter, Receiver, Half Duplex.

\section{Introduction}

In the era of digital communication, data transmission is a common need of every individual to communicate their devices with the remote devices or sometimes nearby devices. Even a common man now is everyday indulged in transferring data in some or the other way like the voice calls, SMS, chats, etc. This is nothing but transmission of data from one end to the other. Some data are securely transferred that should not be readable or writable to public as in the case of defense, bank data, etc. But still there is a vast risk of data being hacked by the anti-social elements. As for far type communications also called Far- field communication requires radio frequency (RF) waves to transmit data over long distance where Personal Area Network is not possible to setup and hence Human Area Network (HAN). But for communication type that is reached within our hands also called as near field communication we can introduce Human Area Network as a medium for transmission of data from start point to end point. Places where data security is must in near field communication. We can replace the system with more adequate and more reliable system called Human Area Network. The idea proposed by T.G. Zimmerman in MIT laboratory in the early 1990s can be implemented now giving a new way for communication world and more dedicated and secured connection[1]. Though we have other techniques like Wi-Fi and Bluetooth for short range or as said near field communication or short range communication the problem still exist with it in crowded places where the throughput is ultimately reduced due to collision of packets. Even the signal in the air can be intercepted with some misuse. Even the strict line of sight direction based activity of Infrared Data Association (IrDA) makes it not appropriate to be used here. Hence, as a result of these problems only a better network type can solve this problem and that can be Human Area Network.

\section{Literature Survey}

All the paper presents an innovative architecture for hardware architecture for networking. As electronic devices become smaller, lower in power requirements, and less expensive, we have begun to adorn our bodies with personal information and communication appliances. Such devices include cellular phones, personal digital assistants (PDA's), pagers and many more. Currently there is no method for these devices to share data.

J. Arun Prakash," Red Tacton: An Innovative Human Area Networking Technology", describes Networking these devices can reduce functional I/O redundancies and allow new conveniences and services. Red Tacton was introduced by Nippon Telegraph and Telephone Corporation (NTT). Red Tacton is a breakthrough technology that uses the surface of the human body as a safe, high speed network transmission path. So we, in this paper are explaining the unique new functional features and enormous potential of Red Tacton as a Human Area networking technology. In this paper I have described features, applications advantages, disadvantages of RedTacton. Levels of connectivity: Wide Area Networks (WAN), Local Area Networks(LAN), and Human Area Networks(HAN) for connectivity to personal information, media and communication appliances within the much smaller sphere of ordinary daily activities.

Sugandha Gupta, Yashu Ahuja," Review of Red Tacton: Evolving Authorizations". It describes, the ideal search engine would be able to match the search queries to the exact context and return results within that context. While Google, Yahoo and Live continue to hold sway in search, here are the engines that take a semantics (meaning) based approach, the end result being more relevant search results which are based on the semantics and meaning of the query Semantic search seeks to improve search accuracy by understanding searcher intent and the contextual meaning of terms. Rather than using ranking algorithms such as Google's PageRank to predict relevancy, Semantic Search uses semantics, or the science of meaning in language, to produce highly relevant search results. In most cases, the goal is to deliver the information queried by a user rather than have a user sort through a list of loosely related keyword results. 


\section{International Journal of Science and Research (IJSR) \\ ISSN (Online): 2319-7064}

Index Copernicus Value (2013): 6.14 | Impact Factor (2014): 5.611

Abdeslem D., Sidi Mohammed, "A New Measure of the Calculation of Semantic Distance between Ontology Concepts", It describes There is a substantial trend in the evolution of online search as people become more sophisticated in their knowledge of search engines and learn that more specific search terms (usually longer search phrases) deliver more accurate search results and in simple search user does not get that record or data which he want. Also the semantic web is to make the meaning of information explicit through semantic mark-up, thus enabling more effective access to knowledge contained in heterogeneous information environments, such as the web. Semantic search plays an important role in realizing this goal, as it promises to produce precise answers to user's queries by taking advantage of the availability of explicit semantics of information. For example, when searching for news stories about Phd students, with traditional searching technologies, we often could only get news entries in which the term Phd students appears.

Jaap Kamps, Maarten Marx, Robert J. Mokken, Maarten de Rijke, "Using WordNet to Measure Semantic Orientations of Adjectives", The semantic web is the idea of teaching the web which is to say teaching the next generation of web search engines and web browsers how to understand the content rather than just the structure on the web. It is teaching the engines to read, understand, draw out the essence and be able to deliver it to us. A primary use of this would be better search engines, but it also has other uses. Web browsers and search engines would be able to answer simple questions by mining the web for the answers. You might also be able to highlight a phrase in your browser and have it come up with more information about that phrase such as reading an article about horses, highlighting a phrase about the proper care for a horse, and having the browser pull up more information about that subtopic.

\section{System Architecture}

It's important to note that there are two different approaches to creating a semantic search engine and a semantic web. One approach is for websites to 'tag' their content, in essence telling search engines what the content is about like a miniature book report about the web page. Another approach is for the engine to be sophisticated enough to scan through the document and figure it out on their own. Of course, this is easier said than done. The biggest fault with the idea of 'tagging' content to give search engines a hint to the content's subject is that it begs to be abused. It is a great system if everyone is using it honestly, but there are a lot of snake oil salesmen out on the web who would use any means possible to get you to their sites. But these same sites already do the same, pulling out all the stops to trick search engines into thinking their keyword phrase is the best, so we might just be par for the course on that one.

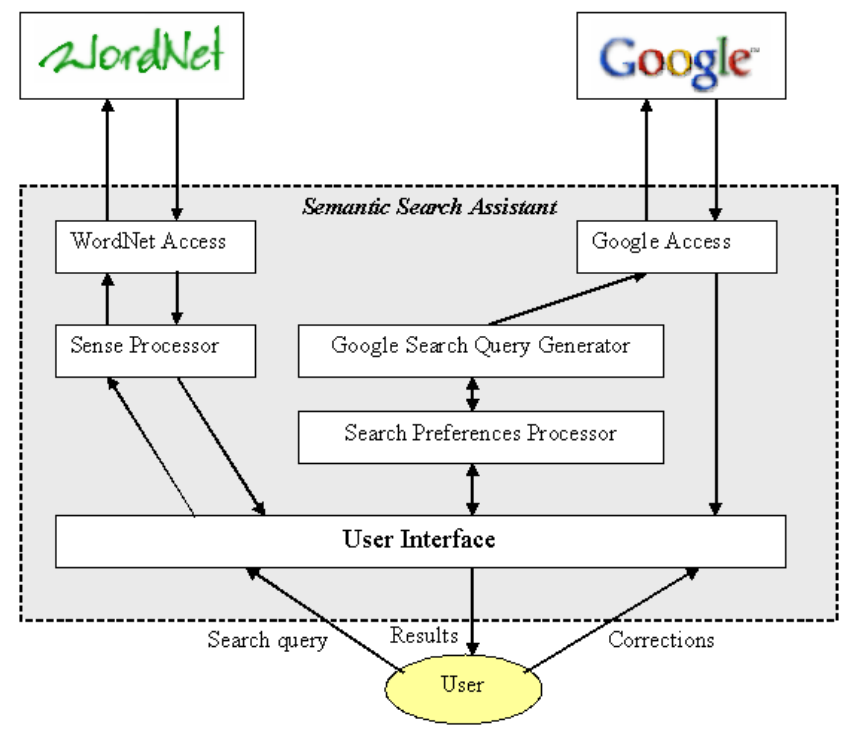

Figure 1: Semantic Search Engine Works

\subsection{The Antonym Dictionary For Review Reversion}

We noticed that DSA highly depends on an external antonym dictionary for review reversion.

\section{1 .1 The Lexicon-based Antonym Dictionary}

An example of lexicon based Antonym dictionary is WordNet. WordNet is a lexical database which groups English words into sets of synonyms called synsets, provides short, general definitions, and records the various semantic relations between these sets. Using the antonym thesaurus it is possible to obtain the words and their opposites. The WordNet antonym dictionary is simple and direct. However, in many languages other than English, such an antonym dictionary may not be readily available. Even if we can get an antonym dictionary, it is still hard to guarantee vocabularies in the dictionary are domain consistent with our tasks. To solve this problem, we furthermore develop a corpus- based method to construct a pseudo-antonym dictionary.

\section{References}

[1] Abdeslem DENNAI, and Sidi Mohammed BENSLIMANE, "A New Measure of the Calculation of Semantic Distance between Ontology Concepts," EEDIS Laboratory, Djillali Liabes University, Sidi Bel Abbes Algeria, vol. 11(2015), pp. 48-56, July. 2015.

[2] Atul Dubey, M. Silvina Tomassone, "Semantic distance in WordNet: An experimental, application-oriented evaluation of five measures", Department of Computer Science, University of Toronto, Toronto, Ontario, Canada Feb. 2014.

[3] Daniel Fried, and Kevin Duh, "INCORPORATING BOTH DISTRIBUTIONAL AND RELATIONAL SEMANTICS IN WORD REPRESENTATIONS", Department of Computer Science, University of Arizona, Tucson, Arizona, USA, Sep. 24, 2014.

[4] Jaap Kamps, Maarten Marx, Robert J. Mokken, Maarten de Rijke, "Using WordNet to Measure Semantic Orientations of Adjectives," Language and Inference 


\section{International Journal of Science and Research (IJSR) \\ ISSN (Online): 2319-7064}

Index Copernicus Value (2013): 6.14 | Impact Factor (2014): 5.611

Technology Group, ILLC, University of Amsterdam., ISSN 1424-8220, vol. 15, no. 3, pp. 311313,Mar. 2008.

[5] Julien Subercaze, Christophe Gravier, Frederique Laforest, "On metric embedding for boosting semantic similarity computations," Universite de Lyon, F-42023, Saint-Etienne, France., vol. 16, no. 3, pp. 311313, 22 Jun 2015.

[6] Vanessa Ayala-Rivera, Patrick McDonagh, Thomas Cerqueus, and Liam Murphy," Ontology-Based Quality Evaluation of Value Generalization Hierarchies for Data Anonymization," School of Computer Science and Informatics, University College Dublin, ISSN 14248220, vol. 17, no. 9, pp. 31846, Mar. 2008.

[7] Andrea Ballatore, Michela Bertolotto and David C. Wilson, "A Structural-Lexical Measure of Semantic Similarity for Geo-Knowledge Graphs," University of California, Santa Barbara, CA 93106, USA, ISSN 1501, vol. 19, no. 3, April. 2015.

\section{Author Profile}

Mr. Rahul Shirbhate is pursuing his Masters of Engineering in the Computer Engineering Department, Sinhgad School of Engineering, and Savitribai Phule University Pune. He received Bachelor of Technology degree in Information Technology from Govt. College of Engineering, University of Amravati, Maharashtra, India.

Prof. Vishal Mogal is the Asst. Professor of Computer Dept. at RMD SSOE College, Pune, having more than 5+ years of experience in the field of teaching and research. The domains of his research are Software Testing, Software Engineering and Web Security. 